\title{
Application of artificial intelligence in the identification of dental implant systems: a literature review
}

\author{
Ho-Kyung Lim, Yeh-Jin Kwon, Eui-Seok Lee \\ Department of Oral and Maxillofacial Surgery, Korea University Guro Hospital, Seoul, Korea
}

\begin{abstract}
Artificial intelligence (Al) can be used across multiple disciplines and has also found application in the dental field. Although there have not been many studies on Al-related to dental implants, identification of implant systems in radiographic images using Al is being actively researched. The purpose of this study was to review articles related to the application of Al for the identification of dental implant systems. A systematic review was conducted using the Pubmed and Scopus databases to identify English language articles about Al and dental implant systems. Factors such as implant systems, data modality, sample size, training time, validation method, Al method, accuracy, sensitivity, specificity, and area under the receiver operating characteristic curve (AUC) were extracted from the 4 selected articles. All the surveyed research adopted pre-trained convolutional neural networks as the Al method. The accuracy of $\mathrm{Al}$ in finding the correct implant system ranged from 51 to 99.5 percent. (JOURNAL OF DENTAL IMPLANT RESEARCH 2020;39(4):48-52)
\end{abstract}

Key Words: Artificial intelligence, Machine learning, Deep learning, Dental implant

\section{INTRODUCTION}

The concept of a strong artificial intelligence (AI) which is capable of mimicking the intelligence of humans has led to, the application of weak AI capable of answering specific questions or solving some disputes ${ }^{1)}$. The concept of weak AI explores different methods for constructing algorithms which learns from a set of data and make predictions. Machine learning aids in developing algorithms supervised by data ${ }^{2)}$. Neural network $(\mathrm{NN})$ was the earliest types of $\mathrm{AI}$ to be built consisting of nodes and weights ${ }^{2}$. The training data attributes influences the computing skills of the network, allowing it to update the connections weight ${ }^{2}$. A simple network structure involving a few layers is called a "shallow" learning neural networks, while a "deep" learning neural network structure involves a plentiful and large layers ${ }^{3}$. Convolutional neural network $(\mathrm{CNN})$ which is a deep learning structure, can be used for extracting many features from abstract filter layers. CNN are commonly useful for processing large and complex figures ${ }^{4}$. The progression made in self-learning back-propagation algorithms has accelerated deep learning by refining the outcome from the data, thereby increasing the computing capability. The advancements in AI today have made it possible to be implemented for solving real-life problems and is applicable in various fields of study ${ }^{5}$.

Since dental implants were first introduced in the 1960s, they have now become the standard treatment method for replacing edentulous area. There are many implant manufacturers, and there are subtle differences in treatment protocols for each company. Jokstad et al. reported that approximately 220 implant brands from 80 companies exist worldwide ${ }^{6}$. If mechanical complications such as screw loosening or screw fracture occur in the implant, accessories provided by the manufacturer should be used to overcome the complications. However, it may be difficult for certain dentists to identify new implant systems simply by only viewing the images of the fixtures in radiographs. Therefore, radiographic identi-

Received November 11, 2020, Revised November 16, 2020, Accepted November 17, 2020.

(c) Journal of Dental Implant Research.

(i) This is an open access article distributed under the terms of the Creative Commons Attribution Non-Commercial License (http://creativecommons.org/licenses/by-nc/4.0) which permits unrestricted non-commercial use, distribution, and reproduction in any medium, provided the original work is properly cited.

Correspondence to: Ho-Kyung Lim, Department of Oral and Maxillofacial Surgery, Korea University Guro Hospital, 148 Gurodong-ro, Guro-gu, Seoul 08308, Korea. Tel: +82-2-2626-1520, Fax: +82-2-837-6245, E-mail: ungassi@naver.com 
fication of implants is especially important to provide appropriate diagnoses and treatments to patients.

For this reason, research to identify implant systems on radiographic images through AI is currently being actively conducted. In this article, papers about AI applied to find and detect dental implant system using dental plain radiography were reviewed.

\section{MATERIALS AND METHODS}

\section{Search strategy}

In PubMed and Scopus, a search was performed for 'Machine learning OR deep learning OR neural network' and 'dental implants AND (identification OR detection OR classification)' until September 2020, and 7, 7, and 9 search results were obtained, respectively. A total of 4 peer-reviewed papers were obtained by removing articles not written in English, those focusing on radiological artifact, papers not related to dental implant, as well as reviews, editorials, and letters.

\section{Data extraction}

The following items were investigated within each paper; Author and year, Implant system, Data modality, Sample Size, Training time, Validation method, AI method, Accuracy, Sensitivity, Specificity, and Area under the receiver operating characteristic curve (AUC).

Among the items listed, Accuracy, Sensitivity, Specificity, AUC are defined as follows.

$$
\begin{aligned}
& \operatorname{Accuracy}(\%)=\frac{\mathrm{TP}+\mathrm{TN}}{\mathrm{TP}+\mathrm{FP}+\mathrm{FN}+\mathrm{TN}} \times 100 \\
& \text { Sensitivity }(\%)=\frac{\mathrm{TP}}{\mathrm{TP}+\mathrm{FN}} \times 100
\end{aligned}
$$

$$
\text { Specificity }(\%)=\frac{\mathrm{TN}}{\mathrm{TN}+\mathrm{FP}} \times 100
$$

TP: True positive, TN: True negative, FP: False positive, FN: False negative.

ROC curve: a curve that visualizes how the evaluation value changes according to the change of each threshold after placing the sensitivity on the $y$-axis and (1-specificity) on the x-axis.

AUC: The value by obtaining the area of the lower area of the ROC curve has a value between $0 \sim 1$, and a higher value means better classification performance.

\section{RESULTS}

The summary of the data derived from the selected papers is given in Table 1.

In all studies, CNN was adopted and used as a main network component. The size of the data set was different for each paper, and it ranged from 801 to 10770. In order to maximize the learning effect of the computer, the number of original data was replicated and increased through cross-validation. Then, deep learning was conducted on more replicated data. In the papers, the frequency of learning was set between 500 and 1000 times. Most of them used pretrained networks such as Alexnet, VGG, GoogLeNet, and Inception in existing servers. For radiographs, two-dimensional images such as panorama or periapical view were used alone or in combination. In the case of implant systems, global-market-leading products such as Straumann and Nobel Biocare were commonly investigated in all paper. Additionally, Osstem and Dentium's products were investigated in a Korean au-

\begin{tabular}{|c|c|c|c|c|c|c|c|c|c|c|}
\hline $\begin{array}{l}\text { Author and } \\
\text { year }\end{array}$ & Implant system & $\begin{array}{l}\text { Data } \\
\text { modality }\end{array}$ & $\begin{array}{l}\text { Sample } \\
\text { size }\end{array}$ & Training & $\begin{array}{l}\text { Validation } \\
\text { method }\end{array}$ & Al method & $\begin{array}{l}\text { Accuracy } \\
(\%)\end{array}$ & $\begin{array}{c}\text { Sensitivity } \\
(\%)\end{array}$ & $\begin{array}{l}\text { Specificity } \\
(\%)\end{array}$ & AUC \\
\hline $\begin{array}{l}\text { Lee et al. } \\
2020\end{array}$ & $\begin{array}{l}3 \text { types (Osstem TSIII, Dentium } \\
\text { Superline, Straumann Bone level) }\end{array}$ & $\begin{array}{l}\text { Panorama, } \\
\text { periapical }\end{array}$ & 10770 & 1000 epoch & $\begin{array}{l}\text { 10-fold } \\
\text { cross-validation }\end{array}$ & $\begin{array}{l}\text { Pretrained CNN } \\
\text { (GoogLeNet Inception-v3) }\end{array}$ & 99.5 & 95.3 & 97.6 & 0.971 \\
\hline $\begin{array}{l}\text { Takahashi et } \\
\text { al. }^{25)} 2020\end{array}$ & $\begin{array}{l}6 \text { types (Nobel Biocare MK } \\
\text { III/IIIG/MKIV/SG, Starumann Bone } \\
\text { level, GC Genesio) }\end{array}$ & Panorama & 1282 & 1000 epoch & & Pretrained CNN (YOLO) & $51 \sim 85$ & $50 \sim 82$ & & 0.72 \\
\hline $\begin{array}{l}\text { Kim et al. }{ }^{26)} \\
2020\end{array}$ & $\begin{array}{l}4 \text { types (Nobel Biocare MK TiUnite, } \\
\text { Dentium Implantium, Straumann } \\
\text { Bone level/Tissue level) }\end{array}$ & Periapical & 801 & 500 epoch & $\begin{array}{l}k \text {-fold } \\
\text { cross-validation }\end{array}$ & $\begin{array}{l}\text { Pretrained CNN } \\
\text { (SqueezeNet, GoogLeNet, } \\
\text { ResNet-18, MobileNet-v2, } \\
\text { and ResNet-50) }\end{array}$ & $93 \sim 98$ & $92 \sim 98$ & $94 \sim 98$ & \\
\hline $\begin{array}{l}\text { Sukegawa et } \\
\text { al. }^{27)} 2020\end{array}$ & $\begin{array}{l}11 \text { types (Zimmer Full Osseotite, } \\
\text { Dentsply Astra EV/TX/Microthread, } \\
\text { Nobel Biocare MKIII/SG/CC, Kyocera } \\
\text { Finesia, Straumann Tissue level) }\end{array}$ & Panorama & 8859 & 700 epoch & $\begin{array}{l}\text { 4-fold } \\
\text { cross-validation }\end{array}$ & $\begin{array}{l}\text { Pretrained CNN (VGG16 } \\
\text { and VGG19) }\end{array}$ & $\begin{array}{c}86.0 \sim \\
93.5\end{array}$ & $\begin{array}{c}84.2 \sim \\
92.8\end{array}$ & $\begin{array}{c}80.2 \sim \\
90.7\end{array}$ & $\begin{array}{c}0.958 \sim \\
1.000\end{array}$ \\
\hline
\end{tabular}
thor's paper, and Zimmer, Astra, GC, and Kyocera's

Table 1. Summary of artificial intelligence articles in identification of dental implants system 
products were investigated in a Japanese author's paper. The accuracy of AI was 51 99.5\%, and the AUC ranged from $0.72 \sim 1.00$.

\section{DISCUSSION}

AI have been studied in various fields within dentistry. Most of them are machine or deep learning using radiographic images or clinical pictures. In the field of oral and maxillofacial surgery, studies have been reported to classify tongue muscles or to provide anatomical guides for segmenting the mandibular canal using $\mathrm{Al}^{7,8)}$. In addition, studies to diagnose oral cancer using tissue slides or cytology images have been reported ${ }^{9)}$. There was also a study analyzing the accuracy of automatic diagnosis using ultrasonography in patients with Sjogren syndrome ${ }^{10)}$. Additionally, there have been studies on the correlation between TMD and TMJ changes on CBCT or MRI ${ }^{11)}$. As for orthodontics, studies on automating measurement points in lateral cephalometry or cone-beam CT have been reported ${ }^{12-14)}$. On top of that, studies have been reported to discriminate the dentition on which orthodontic treatment was performed, or to analyze the relationship between age and face ${ }^{15,16)}$. In cariology, studies to detect dental caries from radiographic or infrared images have been conducted ${ }^{17,18)}$. Also, studies to find normal root morphology, root fracture, and periapical pathogen have also been reported ${ }^{19-21)}$. In prosthodontics, there have been studies to distinguish between restorations and normal teeth in clinical pictures, or to predict the color tone of porcelain powder or composite resin ${ }^{22,23)}$.

Researches on dental implants using AI had yet to be conducted only on the determining the implant system. The reason that only studies on implant systems have been conducted is thought that AI research using radiographic images or clinical images is relatively easy to perform. However, clinicians' desperate need for grasping the implant system is probably the main reason. When a patient has undergone an implant procedure at another hospital, or when the clinical information is not available because of an implant treatment long ago, clinicians must infer the implant system only by looking at radiographs. As mentioned in the introduction, when prosthetic complications of implants occur, clinicians need to know the implant system to solve the complications. Considering these issues, an AI based approach seems to be a potentially suitable solution to solve that problem, and most studies were conducted to focus on developing an automated identification system of implants from radiographic images using object detection.

The accuracy of AI was 51 99.5\%, and a large difference in accuracy was observed between literatures. Takahashi et al.'s research showed relatively low accuracy of $51 \sim 85 \%$, while other papers had an accuracy of $90 \%$ or more, and Kim's research showed that the accuracy was 93 98\% even though the sample size was only $801^{24-27}$. Presumably, this is likely to be the effect of sample size replication by cross-validation. In the study of Takahashi et al., the content of cross-validation was not mentioned ${ }^{25)}$. Even though the sample size was not small, as a result, accuracy may be low because only a small scale of learning was performed.

Currently, the areas that AI must overcome are largely classified into four categories. The first is data acquisition. Establishing an open-access standard data set will play a more important role since the sample size is ultimately directly related to accuracy ${ }^{28)}$. The second is interpretability. Data-based AI cannot explain the decision-making process because it uses a purely computational method, so it needs to be supplemented to compensate mistake ${ }^{29)}$. The third is Computing Power. As the AI model becomes more complex, the output speed depends on the computing power of the computer, so the advancement of hardware must be accompanied ${ }^{30)}$. The last is Ethical consideration. Since AI does not imply standards on human ethics, development with ethical issues must be done for symbiosis with humans ${ }^{31}$.

Contemporary AI excels in utilizing formalized knowledge and extracting information from massive data sets. However, it is hard to involve human emotion in its algorithm. AI should be viewed as an augmentation tool and, at times, relieve dentists so that they can perform more valued tasks, such as integrating emotional sympathy of patient and improving dentist-patient interactions $^{32)}$. In dental implantology, where humans are currently in charge of a large part, it is expected that more development will be achieved through the help of AI in the near future. 


\section{CONFLICT OF INTEREST}

No potential conflict of interest relevant to this article was reported.

\section{REFERENCES}

1. Park WJ, Park JB. History and application of artificial neural networks in dentistry. Eur J Dent 2018;12:594-601.

2. Mupparapu M, Wu CW, Chen YC. Artificial intelligence, machine learning, neural networks, and deep learning: futuristic concepts for new dental diagnosis. Quintessence Int 2018;49:687-8.

3. Hwang JJ, Jung YH, Cho BH, Heo MS. An overview of deep learning in the field of dentistry. Imaging Sci Dent 2019;49:1-7.

4. Shan T, Tay FR, Gu L. Application of Artificial Intelligence in Dentistry. J Dent Res 2020:22034520969115.

5. Shahid N, Rappon T, Berta W. Applications of artificial neural networks in health care organizational decision-making: a scoping review. PLoS One 2019;14:e0212356.

6. Jokstad A, Braegger U, Brunski JB, Carr AB, Naert I, Wennerberg A. Quality of dental implants. Int Dent J 2003;53(6 Suppl 2):409-43.

7. Alabi RO, Elmusrati M, Sawazaki-Calone I, Kowalski LP, Haglund C, Coletta RD, et al. Comparison of supervised machine learning classification techniques in prediction of locoregional recurrences in early oral tongue cancer. Int J Med Inform 2020;136:104068.

8. Gerlach NL, Meijer GJ, Kroon DJ, Bronkhorst EM, Berge SJ, Maal TJ. Evaluation of the potential of automatic segmentation of the mandibular canal using cone-beam computed tomography. Br J Oral Maxillofac Surg 2014;52:838-44.

9. Sunny S, Baby A, James BL, Balaji D, N VA, Rana MH, et al. A smart tele-cytology point-of-care platform for oral cancer screening. PLoS One 2019;14:e0224885.

10. Kise Y, Shimizu M, Ikeda H, Fujii T, Kuwada C, Nishiyama M, et al. Usefulness of a deep learning system for diagnosing Sjogren's syndrome using ultrasonography images. Dentomaxillofac Radiol 2020;49:20190348.

11. Shoukri B, Prieto JC, Ruellas A, Yatabe M, Sugai J, Styner M, et al. Minimally invasive approach for diagnosing TMJ osteoarthritis. J Dent Res 2019;98:1103-11.

12. Kok H, Acilar AM, Izgi MS. Usage and comparison of artificial intelligence algorithms for determination of growth and development by cervical vertebrae stages in orthodontics. Prog Orthod 2019;20:41.

13. Amasya H, Yildirim D, Aydogan T, Kemaloglu N, Orhan K. Cervical vertebral maturation assessment on lateral cephalometric radiographs using artificial intelligence: comparison of machine learning classifier models. Dentomaxillofac Radiol 2020;49:20190441.

14. Gupta A, Kharbanda OP, Sardana V, Balachandran R, Sardana HK. A knowledge-based algorithm for automatic detection of cephalometric landmarks on CBCT images. Int J Comput Assist
Radiol Surg 2015;10:1737-52.

15. Patcas R, Bernini DA, Volokitin A, Agustsson E, Rothe R, Timofte R. Applying artificial intelligence to assess the impact of orthognathic treatment on facial attractiveness and estimated age. Int J Oral Maxillofac Surg 2019;48:77-83.

16. Wang X, Cai B, Cao Y, Zhou C, Yang L, Liu R, et al. Objective method for evaluating orthodontic treatment from the lay perspective: An eye-tracking study. Am J Orthod Dentofacial Orthop 2016;150:601-10.

17. Casalegno F, Newton T, Daher R, Abdelaziz M, Lodi-Rizzini A, Schurmann F, et al. Caries detection with near-infrared transillumination using deep learning. J Dent Res 2019;98: 1227-33.

18. Schwendicke F, Elhennawy K, Paris S, Friebertshauser P, Krois $J$. Deep learning for caries lesion detection in near-infrared light transillumination images: a pilot study. J Dent 2020;92: 103260.

19. Setzer FC, Shi KJ, Zhang Z, Yan H, Yoon H, Mupparapu M, et al. Artificial intelligence for the computer-aided detection of periapical lesions in cone-beam computed tomographic images. J Endod 2020;46:987-93.

20. Orhan K, Bayrakdar IS, Ezhov M, Kravtsov A, Ozyurek T. Evaluation of artificial intelligence for detecting periapical pathosis on cone-beam computed tomography scans. Int Endod J 2020;53:680-9.

21. Hiraiwa T, Ariji Y, Fukuda M, Kise Y, Nakata K, Katsumata A, et al. A deep-learning artificial intelligence system for assessment of root morphology of the mandibular first molar on panoramic radiography. Dentomaxillofac Radiol 2019;48: 20180218.

22. Yamaguchi S, Lee C, Karaer O, Ban S, Mine A, Imazato S. Predicting the debonding of CAD/CAM composite resin crowns with Al. J Dent Res 2019;98:1234-8.

23. Yoneda M, Yamada K, Izumi T, Matsuzaki E, Maruta M, Hatakeyama J, et al. Development of an error-detection examination for conservative dentistry education. Clin Exp Dent Res 2020;6:69-74.

24. Lee JH, Jeong SN. Efficacy of deep convolutional neural network algorithm for the identification and classification of dental implant systems, using panoramic and periapical radiographs: A pilot study. Medicine (Baltimore) 2020;99:e20787.

25. Takahashi T, Nozaki K, Gonda T, Mameno T, Wada M, Ikebe K. Identification of dental implants using deep learning-pilot study. Int J Implant Dent 2020;6:53.

26. Kim JE, Nam NE, Shim JS, Jung YH, Cho BH, Hwang JJ. Transfer learning via deep neural networks for implant fixture system classification using periapical radiographs. J Clin Med 2020;9.

27. Sukegawa S, Yoshii K, Hara T, Yamashita K, Nakano K, Yamamoto N, et al. Deep neural networks for dental implant system classification. Biomolecules 2020;10.

28. Hosny A, Parmar C, Quackenbush J, Schwartz LH, Aerts H. Artificial intelligence in radiology. Nat Rev Cancer 2018;18: 500-10.

29. Kulikowski CA. Beginnings of artificial intelligence in medicine (aim): computational artifice assisting scientific inquiry and clinical art - with reflections on present aim challenges. Yearb 
52 Lim HK, et al: Application of artificial intelligence in identification of dental implants system: literature review

Med Inform 2019;28:249-56.

30. Fabrizio A, Meyer B, Fabregat R, Corminboeuf C. Quantum chemistry meets machine learning. Chimia (Aarau) 2019;73: 983-9.

31. Currie G, Hawk KE, Rohren EM. Ethical principles for the appli- cation of artificial intelligence (Al) in nuclear medicine. Eur J Nucl Med Mol Imaging 2020;47:748-52.

32. Recht M, Bryan RN. Artificial intelligence: threat or boon to radiologists? J Am Coll Radiol 2017;14:1476-80. 